\title{
Creation Field Teoride Yüksek Boyutlu Kaluza-Klein Uzay-Zamanı İçin Bazı Topolojik Kusurlar
}

\author{
Nur Gültekin*, Can Aktaş \\ Çanakkale Onsekiz Mart Üniversitesi, Fen Edebiyat Fakültesi, \\ Matematik ABD
}

\section{Özet}

Evrenin oluşumu ile ilgili en tutarlı teorilerden biri Büyük Patlama (Big Bang) kuramıdır. Bu kurama göre, evren 13,8 milyar yıl önce çok yoğun ve sicak bir noktadan patlayarak oluşmuştur. Büyük patlama teorisine göre Planck dönemi olarak da adlandırılan $t \rightarrow 10^{-43}$ sn yani evrenin başlangıcında dört temel kuvvet (kütle çekim, elektromanyetik, kuvvetli ve zayıf kuvvet) bir arada bulunmaktaydı. Daha sonra bu dört temel kuvvet birbirinden ayrılmaya başlamıştır. Önce kütle çekim kuvveti diğer üç kuvvetten ayrılmıştır. Sonrasında ise kuvvetli kuvvet diğer kuvvetlerden ayrılmıştır. Son olarak da elektromanyetik ve zayıf kuvvetler birbirinden ayrılmıştır. Bu kuvvetlerin birbirinden ayrılması sırasında evren yüksek sıcaklık evresinden düşük sıcaklık evresine geçmiș ve bu sırada evrende simetri bozulmaları meydana gelmiștir. Bu simetri bozulmaları evrende çeşitli topolojik kusurlar oluşmasına neden olmuştur. Bu simetri bozulmalarında sıfır boyutlu monopoller ve bir boyutlu sicimler oluşmuştur. Ayrık simetrinin bozulması durumunda ise iki boyutlu domain wall'lar oluşmuştur.

Son yıllarda yapılan birçok çalışma ve gözlem evrenin ilk durumundan günümüze ivmelenerek genişlediğini göstermektedir. Bilim adamları ivmelenmenin ve genişlemenin nedenlerini açıklayabilmek için çeşitli teoriler ortaya koymuşlardır. Bu ivmelenme ve genişlemeye karanlık madde ve karanlık enerji gibi maddelerin neden olabileceği düşünülmektedir. Ayrıca, bu ivmelenme ve genişleme, Lyra, Creation Field, Brans-Dicke, $f(R), f(R, T)$ gibi bazı alternatif gravitasyon teorileriyle de açıklanabilmektedir.

Creation field teorisi kütlesi ve yükü olmayan skaler alanda maddenin oluştuğunu ileri süren alternatif gravitasyon teorilerinden biridir. Bu teori ile büyük patlama teorisinde karşılaştığımız düzlük ve ufuk problemleri çözülmeye çalışılmıştır.

Bu çalışmada evrenin ilk anlarında simetri bozulmaları esnasında ortaya çıkan topolojik kusurlardan biri olan Domain Wall madde alınarak yüksek boyutlu Kaluza -Klein metriği için Creation Field teoride Einstein alan denklemleri elde edilmiştir. Bu denklemlerin çözümü için evrenin ivmelenmesinde önemli bir nicelik olan frenleme parametresi doğrusal ve sabit formda alınmıştır. Bu çözümler geometrik ve fiziksel olarak detaylı şekilde incelenmiştir. Bunun yanı 
sıra, kinematik nicelikler elde edilmiş ve fiziksel olarak yorumlanmıştır. Ayrıca, yüksek boyutların çözümler üzerindeki etki ve genellemeleri ile ivmelenme problemi çözülmeye çalışılacaktır.

Anahtar Sözcükler: creation field teori, domain wall, sabit frenleme parametresi, topolojik kusurlar, yüksek boyutlu kaluza-klein uzay-zamanı

\title{
Some Topological Defects in Creation Field Theory for Kaluza-Klein Universe
}

\begin{abstract}
:
One of the most consistent theories about the formation of the universe is the Big Bang theory. According to the Big Bang theory, the universe was formed 13.8 billion years ago from a very dense and hot point. According to theory of the Big Bang, there were four basic forces (gravity, electromagnetic, strong and weak forces) in the beginning of the universe called the Planck period. Then these four basic forces began to separate. Firstly, the gravitational force was separated from the other three forces. Then the strong force was divided from the other forces. Finally, the electromagnetic and weak forces were separated from each other. During the separation of these forces, the universe has gone from a high temperature to a low temperature, with symmetry breaking in the universe. These symmetry breaking has led to the formation of topological defects in the universe. In this symmetry breaking, zero-dimensional monopoles and one-dimensional strings were formed. In the case of discrete symmetry, two-dimensional Domain Walls were formed.
\end{abstract}

In recent years, observations made show that the universe is accelerating expansion. Scientists have put forth several theories to explain the reasons for acceleration and the expansion. It is thought that acceleration and expansion can cause materials such as dark matter and dark energy. This acceleration and expansion can also be explained by some alternative gravitation theories such as Lyra, Creation Field, Brans-Dicke, $\mathrm{f}(\mathrm{R}), \mathrm{f}(\mathrm{R}, \mathrm{T})$.

Creation Field theory is one of the alternative gravitation theories in chargeless and massless scalar field for the formation of the matter. With this theory, we tried to solve the flatness and horizon problems that we encountered in the Big Bang theory.

In this study, Einstein field equations were obtained for the Creation Field theory for the highdimensional Kaluza-Klein metric by taking Domain Wall matter, one of the topological defects that occurred during symmetry breaking in the first moments of the universe. The deceleration parameter, which is an important quantity in the acceleration of the universe, is taken in linear and constant form for the solution of these equations. These solutions were analyzed in detail as geometric and physical. Furthermore, kinematic quantities obtained and physically was interpreted. In addition, the problem will be resolved with the effects of acceleration and generalization of the solutions of the high dimensions.

Keywords: creation-field theory, domain wall, constant deceleration parameter, topological defects, high dimensional kaluza-klein metric 


\section{Giriș}

Birçok kozmolojik gözlem evrenimizin ivmelenerek genişlediğini göstermektedir (Efstathion ve ark., 2002; Tegmark ve ark., 2004). Bilim adamları bu ivmelenmenin ve genişlemenin nedenlerini açıklayabilmek amacıyla çeşitli teoriler ileri sürmüşlerdir. İleri sürdükleri teorilerden biri bu genişleme ve ivmelenmeye karanlık madde ve karanlık enerjinin sebep olabileceği görüşüdür. Bilindiği gibi evrenin \%4'lük kısmı bugün bildiğimiz maddeden, \%73'lük kısmı karanlık enerjiden ve \%23'lük kısmı da karanlık maddeden oluşmaktadır. Ayrıca bilim adamları bu genişleme ve ivmelenmeyi açıklayabilmek için Einstein'ın 1915 yılında ortaya attığı alan denklemlerini modifiye ederek çeşitli alternatif gravitasyon teorileri ileri sürmüşlerdir. Bu alternatif gravitasyon teorileri arasinda $f(G)$ teori, $f(R)$ teori, Creation Field teori, $f(R, T)$ teori, Lyra teorisi, Brans- Dicke, Self Creation teori vb. sayılabilir.

Kütlesiz ve yüksüz skaler alanda maddenin oluşumunu açıklamaya çalışan Creation field teorisi önemli modifiye teorilerden biridir (Hoyle ve Narlikar, 1966). Einstein'in 1915'de öne sürdüğü genel relativite teorisine alternatif bir teori 1948'de Bondi ve Gold tarafından ileri sürülmüştür (Bondi ve Gold, 1948). 1966 yılında Hoyle ve Narlikar, Bondi ve Gold'un 1948 de ortaya attığ 1 bu teoriyi yeniden düzenlenmişlerdir (Hoyle ve Narlikar, 1966; Bondi ve Gold, 1948).

1966 yılında Hoyle ve Narlikar'ın ileriye sürdüğü teori, Bondi ve Gold'un teorisinden farklı olarak hiçbir Big Bang tipi tekillik bulundurmamaktadır. Creation Field teorisi Büyük Patlama teorisinde karşımıza çıkan düzlemsellik ve ufuk problemlerine çözüm bulmayı amaçlamıştır (Hoyle ve Narlikar,1966; Bondi ve Gold,1948).

Büyük birleşim teorisi evrenin ilk durumunu açıklayabilmeyi amaçlamaktadır. Doğadaki elektromanyetik, gravitasyon, zayıf kuvvet ve güçlü çekirdek kuvvetinin simetri yardımıyla birleştirme fikri olarak bilinen Büyük Birleşim teorisine göre evren ilk anlardaki yüksek sıcaklık döneminden (simetrik evre) düşük sicaklık dönemine (bozuk simetrik evre) geçerken birçok simetri bozulması meydana gelmiştir (Vilenkin ve Shelleard, 1994). Bu simetri bozulmaları evrende çeşitli topolojik kusurların (defect) oluşmasına sebep olmuştur (Vilenkin ve Shelleard, 1994). Bu simetri bozulmalarında faz geçiş niteliği ve maddenin simetri özellikleri tarafından belirlenen farklı çeşitlerde birçok topolojik kusur oluşmuştur: Bunlar iki boyutlu Domain Wall'lar, bir boyutlu sicimler (string'ler) ve sifir boyutlu monopollerdir. (Vilenkin ve Shelleard, 1994). Monopollerin kuzey ve güney şeklinde manyetik yüke sahip küresel simetri bozulduğunda oluşan küp şeklinde kusur oldukları tahmin edilmektedir (Vilenkin ve Shelleard, 1994). Sicimler silindirik simetri bozulduğunda oluşan eksenle ilgili bir boyutlu doğrulardır. Domain Wall'ler, bir ayrık simetri faz geçişi sırasında oluşan iki boyutlu zarlardır. Bu zar duvarlar evreni ayrık hücrelere bölen kapalı hücre duvarlarına benzer (Vilenkin ve Shelleard, 1994). Topolojik kusurlar aşırı derecede yüksek enerji ortamında oluştuklarından dünyadaki fizik deneylerinde üretilmeleri imkânsız gibidir. Ancak evrenin oluşumundan meydana gelen topolojik kusurlar teorik olarak incelenebilir.

Creation Field teorisi son yıllarda birçok bilim adamı tarafından çalışılmıştır. Rahaman ve ark. (2005) küresel simetrik metrik için Creation field teoride global monopol çözümlerini araştırmiştır. Rahaman ve ark. (2007) kurt deliklerinin Creation field teorisinde küresel simetrik metriğinde chapling gaz çözümlerini çalışmışlardır. Adhav ve ark. (2010) Bianchi I metriği için Domain Wall alarak çözüm elde etmişlerdir. 
Ayrıca yine Adhav ve ark.(2011) LRS Bianchi- I metriğinde creation field teoride frenleme parametresini sabit alarak çalışma yapmışlardır. Bali ve ark.(2011) FRW metriğinde creation field teoride kozmolojik sabitli çözümler bulmuşlardır. Adhav ve ark.(2008) Bianchi- I metriğinde sicim madde için creation field teoride alan denklemlerinin çözüm ve sonuçlarını incelemişlerdir. Singh ve ark.(2014) creation field teoride Bianchi III uzay-zamanı için kuark madde çözümlerini irdelemişlerdir.

Bu çalışmada evrenin ilk anlarında ortaya çıkan topolojik kusurlardan biri olan Domain Wall madde alınarak yüksek boyutlu Kaluza -Klein metriği için Creation Field teoride Einstein alan denklemlerinin çözümleri elde edilmiş ve çözümler fiziksel olarak irdelenmiştir.

\section{Materyal ve Metot}

Creation field teorideki modifiye Einstein alan denklemleri

$$
\mathrm{R}_{\mathrm{ik}}-\frac{1}{2} \mathrm{~g}_{\mathrm{ik}} \mathrm{R}=-\left(\mathrm{T}_{\mathrm{ik}}^{\mathrm{m}}+\mathrm{T}_{\mathrm{ik}}^{\mathrm{C}}\right)
$$

şeklindedir. Burada $\mathrm{R}_{\mathrm{ik}}$ Ricci tensörü, $\mathrm{g}_{\mathrm{ik}}$ metrik tensör, R Ricci skaleri, $\mathrm{T}_{\mathrm{ik}}^{\mathrm{m}}$ Einstein teorisindeki enerji momentum tensörü, $\mathrm{T}_{\mathrm{ik}}^{\mathrm{C}}$ Creation field teorisindeki enerji momentum tensörü olup aşağıdaki şekilde tanımlıdır (Adhav ve ark., 2010).

$$
\mathrm{T}_{\mathrm{ik}}^{\mathrm{C}}=\frac{\mathrm{f}}{2} \mathrm{~g}_{\mathrm{ik}} \mathrm{C}^{\mathrm{l}} \mathrm{C}_{\mathrm{l}}-\mathrm{fC}_{\mathrm{i}} \mathrm{C}_{\mathrm{k}}
$$

Burada $C_{\mathrm{i}}=\frac{\partial \mathrm{C}}{\partial \mathrm{x}^{\mathrm{i}}}$ ve $f>0$ sabittir (Adhav ve ark., 2010). Topolojik kusurlardan biri olarak bilinen Domain Wall madde için enerji momentum tensörü aşağıdaki şekildedir.

$$
\mathrm{T}_{\mathrm{ij}}^{\mathrm{m}}=\rho\left(\mathrm{g}_{\mathrm{ij}}+\omega_{\mathrm{i}} \omega_{\mathrm{j}}\right)+\mathrm{p} \omega_{\mathrm{i}} \omega_{\mathrm{j}}
$$

Burada $\omega^{i}=(0,0,0,0, \ldots, 1), \rho$ enerji yoğunluğu ve $p$ basınçtır. Ayrıca

ve

$$
\mathrm{p}=\mathrm{p}_{\mathrm{m}}-\sigma_{\mathrm{w}}
$$

$$
\rho=\rho_{\mathrm{m}}+\sigma_{w}
$$

olup, burada $\rho_{\mathrm{m}}$ Domain Wall maddenin enerji yoğunluğu, $\mathrm{p}_{\mathrm{m}}$ Domain Wall maddenin basıncı ve $\sigma_{w}$ Domain Wwall maddenin gerilimidir (Vilenkin,1981; Katore ve ark., 2016). Domain Wall madde için durum denklemi ise aşağıdaki gibidir.

$$
\mathrm{p}=(\gamma-1) \rho
$$

Burada $\gamma$ bir sabit olup $1 \leq \gamma \leq 2$ dir (Vilenkin, 1981; Katore ve ark., 2016).

\section{Bulgular ve Tartışma}

Yüksek boyutlu anizotropik ve homojen Kaluza - Klein metriği

$$
d s^{2}=-d t^{2}+A^{2}(t)\left(d x_{1}^{2}+\cdots+d x_{n-2}^{2}\right)+B^{2}(t) d x_{n-1}^{2}
$$


şeklindedir (Adhav ve ark., 2008).

(4.1) metriği için kinematik nicelikler; Hacim (V), Hubble parametresi (H), ortalama anizotropi parametresi $(\Delta)$ ve frenleme parametresi $(\mathrm{q})$ aşağıdaki şekilde tanımlıdır.

$$
\begin{aligned}
& \mathrm{V}=\mathrm{R}^{\mathrm{n}-1}=\mathrm{A}^{\mathrm{n}-2} \mathrm{~B} \\
& \mathrm{H}=\frac{\dot{\mathrm{R}}}{\mathrm{R}}=\frac{1}{\mathrm{n}-1}\left[(\mathrm{n}-2) \frac{\dot{\mathrm{A}}}{\mathrm{A}}+\frac{\dot{\mathrm{B}}}{\mathrm{B}}\right] \\
& \Delta=\frac{1}{\mathrm{n}-1} \sum_{\mathrm{i}=1}^{\mathrm{n}-1}\left(\frac{\mathrm{H}_{\mathrm{i}}-\mathrm{H}}{\mathrm{H}}\right)^{2}=\frac{(\mathrm{n}-1)(\mathrm{n}-2)\left(\frac{\dot{\mathrm{A}}}{\mathrm{A}}-\frac{\dot{\mathrm{B}}}{\mathrm{B}}\right)^{2}}{\left[(\mathrm{n}-2) \frac{\dot{A}}{\mathrm{~A}}+\frac{\dot{\mathrm{B}}}{\mathrm{B}}\right]^{2}} \\
& \mathrm{q}=\frac{\mathrm{d}}{\mathrm{dt}}\left(\frac{1}{\mathrm{H}}\right)-1=-\frac{\mathrm{R} \ddot{\mathrm{R}}}{\dot{\mathrm{R}}^{2}}
\end{aligned}
$$

Burada $\mathrm{H}_{1=} \mathrm{H}_{2}=\cdots=\mathrm{H}_{\mathrm{n}-2}=\frac{\dot{\mathrm{A}}}{\mathrm{A}}, \mathrm{H}_{\mathrm{n}-1}=\frac{\dot{\mathrm{B}}}{\mathrm{B}}$ Hubble çarpanlarıdır. (3.1) - (3.3) denklemlerinden yüksek boyutlu Kaluza-Klein metriği için Creation field teorideki Einstein alan denklemleri aşağıdaki gibi elde edilir.

$$
\begin{aligned}
& \frac{(n-3)}{2}\left[\frac{2 \dot{A} \dot{B}}{A B}+(n-4) \frac{\dot{A}^{2}}{A^{2}}+\frac{2 \ddot{A}}{A}\right]+\frac{\ddot{B}}{B}=\frac{1}{2} f \dot{C}^{2}-\rho \\
& \frac{(n-2)}{2}\left[(n-3) \frac{\dot{A}^{2}}{A^{2}}+\frac{2 \ddot{A}}{A}\right]=\frac{1}{2} f \dot{C}^{2}-\rho \\
& \frac{(n-2)}{2}\left[(n-3) \frac{\dot{A}^{2}}{A^{2}}+\frac{2 \dot{A} \dot{B}}{A B}\right]=-\frac{1}{2} f^{2}+p
\end{aligned}
$$

Burada nokta zamana göre türevi ifade etmektedir. Enerji korunum denklemi olan $\mathrm{T}^{\mathrm{ik}}{ }_{\text {k }}=$ 0'dan

$\dot{\rho}+\left[(\mathrm{n}-2) \frac{\dot{\mathrm{A}}}{\mathrm{A}}+\frac{\dot{\mathrm{B}}}{\mathrm{B}}\right](\rho+\mathrm{p})-\mathrm{f} \dot{\mathrm{C}} \ddot{\mathrm{C}}-\dot{\mathrm{fC}}^{2} \frac{\dot{\mathrm{B}}}{\mathrm{B}}-(\mathrm{n}-2) \mathrm{f} \dot{\mathrm{C}}^{2} \frac{\dot{\mathrm{A}}}{\mathrm{A}}=0(4.9)$

elde edilir. Yukarıda elde edilen alan denklemleri((4.6) - (4.8) denklemleri) 5 bilinmeyenli (A, $\mathrm{B}, \mathrm{C}, \mathrm{p}, \rho) 3$ denklemden oluşmaktadır. Bu denklemleri çözebilmemiz için ilave denklemlere ihtiyacımız vardır. İlave denklem olarak genellikle anizotropi parametresi, frenleme parametresi gibi denklemler alınır. Bu çalışmada ilave denklem olarak hem frenleme parametresi hem de ortalama anizotropi parametresi kullanılmıştır. (4.4) denkleminden $\Delta=\xi$ (sbt) denklemi çözülürse

$$
A=b B \frac{-(n-2)(n+\xi-1)+\sqrt{\xi(n-2)(n-1)^{3}}}{(n-2)(n \xi-n-2 \xi+1)}
$$

elde edilir. Burada b sabittir. Genelliği bozmaksızın $b=1$ ve 
$\frac{-(\mathrm{n}-2)(\mathrm{n}+\xi-1)+\sqrt{\xi(\mathrm{n}-2)(\mathrm{n}-1)^{3}}}{(\mathrm{n}-2)(\mathrm{n} \xi-\mathrm{n}-2 \xi+1)}=r$ alabiliriz. Bu durumda (4.10) denklemi

$$
A(t)=B(t)^{r}
$$

şekline dönüşür. Domain Wall madde çözümünde frenleme parametresini aşağıdaki gibi lineer formda alarak çözümleri araştıralım.

$$
\mathrm{q}=-\mathrm{kt}+\mathrm{m}-1
$$

Burada $\mathrm{k} \geq 0$ ve $\mathrm{m} \geq 0$ sabitlerdir (Akarsu ve Dereli, 2012). (4.5) ve (4.12) denklemleri çözülürse $\mathrm{k}$ ve $\mathrm{m}$ sabitlerinin durumlarına göre 2 farklı model ortaya çıkar.

\subsection{Creation Field Teoride $\mathbf{k} \neq \mathbf{0}$ ve $\mathbf{m} \neq \mathbf{0}$ için Domain Wall Madde Çözümü}

(4.5) ve (4.12) denklemlerinden evren yarıçapı aşağıdaki gibi elde edilir.

$$
\mathrm{R}(\mathrm{t})=\mathrm{c}_{2}\left(\frac{\mathrm{kst}-\mathrm{ms}+1}{-\mathrm{kst}+\mathrm{ms}+1}\right)^{\mathrm{s}}
$$

Burada $s=\frac{1}{\sqrt{\mathrm{m}^{2}-2 \mathrm{c}_{1} \mathrm{k}}}=$ sabit, $\mathrm{c}_{1}$ ve $\mathrm{c}_{2}$ integral sabitidir.

(4.6) - (4.8) denklemleri (4.2) ve (4.13) denklemlerinin yardımı ile çözülürse metrik potansiyelleri aşağıdaki şekilde bulunur.

$$
\begin{aligned}
& A(t)=R^{\frac{(n-1) r}{1+(n-2) r}}=\left[\frac{k s t-m s+1}{-k s t+m s+1}\right]^{\frac{s r(n-1)}{1+(n-2) r}} c_{2}^{\frac{s r(n-1)}{1+(n-2) r}} \\
& B(t)=R^{\frac{(n-1)}{1+(n-2) r}}=\left[\frac{k s t-m s+1}{-k s t+m s+1}\right]^{\frac{s(n-1)}{1+(n-2) r}} c_{2}^{\frac{s(n-1)}{1+(n-2) r}}
\end{aligned}
$$

(4.14) ve (4.15) denklemleri (4.6) - (4.8) denklemlerinde yerine yazılır ve birbirinden çıkarılırsa

$$
(r-1)(n-1)(k t-m+n-1) s^{4} k^{2}=0
$$

elde edilir. Bu denklemin sağlaması için $n \geq 4, s \neq 0$ vek $\neq 0$ olduğundan $r=1$ olmalıdır.

$$
\text { (4.6) - (4.8) denklemlerinden Creation field teorideki skaler alan } \mathrm{C}(\mathrm{t}) \text { ise }
$$

$$
C(t)=c_{3} t+c_{4}
$$

şeklindedir. Burada $c_{3}$ ve $c_{4}$ integral sabitleridir. ve basınç

(4.14) ve (4.15) denklemleri (4.6) - (4.8) denklemlerinde yerine yazılırsa enerji yoğunluğu

$$
\rho(t)=\frac{1}{2} c_{3}^{2} f+\frac{2 k^{2} s^{4}(n-1)(n-2)}{(k s t-m s+1)^{2}(k s t-m s-1)^{2}}
$$




$$
p(t)=\frac{1}{2} c_{3}^{2} f+\frac{2 k^{2} s^{4}(n-2)(2 k t+2 m-n+1)}{(k s t-m s+1)^{2}(k s t-m s-1)^{2}}
$$

şeklinde bulunur. (4.18) ve (4.19) denklemleri (3.4) - (3.6) denklemlerinde yerine yazılırsa Domain Wall maddenin enerji yoğunluğu, basınc1 ve gerilimi

$$
\begin{gathered}
\mathrm{p}_{\mathrm{m}}=\frac{\gamma-1}{\gamma}\left(\mathrm{c}_{3}{ }^{2} \mathrm{f}+\frac{4 \mathrm{k}^{2} \mathrm{~s}^{4}(\mathrm{n}-2)(\mathrm{kt}-\mathrm{m})}{(\mathrm{kst}-\mathrm{ms}+1)^{2}(\mathrm{kst}-\mathrm{ms}-1)^{2}}\right) \\
\rho_{\mathrm{m}}=\frac{1}{\gamma}\left(\mathrm{c}_{3}{ }^{2} \mathrm{f}+\frac{4 \mathrm{k}^{2} \mathrm{~s}^{4}(\mathrm{n}-2)(\mathrm{kt}-\mathrm{m})}{(\mathrm{kst}-\mathrm{ms}+1)^{2}(\mathrm{kst}-\mathrm{ms}-1)^{2}}\right) \\
\sigma_{\omega}=\frac{1}{2}(\gamma-2) \mathrm{c}_{3}{ }^{2} \mathrm{f}+\frac{2 \mathrm{k}^{2} \mathrm{~s}^{4}(\gamma-1)(\mathrm{n}-2)(\mathrm{kt}+2 \mathrm{~m}-2 \gamma(\mathrm{n}-1))}{(\mathrm{kst}-\mathrm{ms}+1)^{2}(\mathrm{kst}-\mathrm{ms}-1)^{2}}
\end{gathered}
$$

şeklinde elde edilir.

\subsection{Creation Field Teoride $\mathbf{k}=\mathbf{0}$ ve $\mathbf{m} \neq 0$ için Domain Wall Madde Çözümü}

(4.12) denkleminde $\mathrm{k}=0$ alınırsa frenleme parametresi

$$
\mathrm{q}=\mathrm{m}-1
$$

olarak elde edilir. Bu durumda (4.5) ve (4.23) denklemlerinden evren yarıçapı aşağıdaki gibi elde edilir.

$$
R(t)=\left(m_{5} t+c_{6}\right)^{\frac{1}{m}}
$$

Burada $c_{5}$ ve $c_{6}$ sabitlerdir. (4.6) -(4.8) denklemleri (4.2) ve (4.24) denklemleri yardımıyla çözülürse

$$
\begin{aligned}
& A(t)=R^{\frac{(n-1) r}{1+(n-2) r}}=\left(m c_{5} t+c_{6}\right)^{\frac{(n-1) r}{(1+(n-2) r) m}} \\
& B(t)=R^{\frac{n-1}{1+(n-2) r}}=\left(m c_{5} t+c_{6}\right)^{\frac{n-1}{(1+(n-2) r) m}}
\end{aligned}
$$

metrik potansiyelleri şeklinde elde edilir. Creation field teosindeki skaler alan

$$
C(t)=c_{7} t+c_{8}
$$

şeklinde bulunur. Buradaki $c_{7}$ ve $c_{8}$ integral sabitleridir. (4.25) ve (4.26) denklemleri (4.6) (4.8) alan denklemlerinde yerine yazılırsa enerji yoğunluğu ve basınç aşağıdaki gibi elde edilir.

$$
\begin{aligned}
& \rho(t)=\frac{c_{7}^{2} f}{2}+\frac{c_{4}{ }^{2}(n-2)(2 m-n+1)}{2\left(m c_{5} t+c_{6}\right)^{2}} \\
& p(t)=\frac{c_{7}{ }^{2} f}{2}+\frac{c_{4}{ }^{2}(n-2)(n-1)}{2\left(m c_{5} t+c_{6}\right)^{2}}
\end{aligned}
$$


(3.4) - (3.6), (4.28) ve (4.29) denklemlerinden Domain Wall maddenin enerji yoğunluğu, basıncı ve gerilimi aşağıdaki şekilde bulunur.

$$
\begin{aligned}
& p_{m}=\frac{\gamma-1}{\gamma}\left(c_{7}{ }^{2} f+\frac{(n-2) c_{4}{ }^{2}}{\left(m c_{5} t+c_{6}\right)^{2}}\right) \\
& \rho_{m}=\frac{1}{\gamma}\left(c_{7}{ }^{2} f+\frac{(n-2) c_{4}{ }^{2}}{\left(m c_{5} t+c_{6}\right)^{2}}\right) \\
& \sigma_{w}=\frac{\gamma-2}{2 \gamma}\left(c_{7}{ }^{2} f+\frac{(n-2) c_{5}{ }^{2}[m(\gamma-1)-\gamma(n-1)]}{\left(m c_{5} t+c_{6}\right)^{2}}\right)
\end{aligned}
$$

\section{Sonuç}

Bu çalışmada, evrenin ilk anlarında meydana gelen topolojik kusurlardan biri olan Domain Wall madde çözümleri yüksek boyutlu Kaluza-Klein metriği için Creation field teoride elde edilmiştir. Frenleme parametresi doğrusal formda ve sabit alınarak farklı iki çözüm bulunmuş ve bu çözümlerin fiziksel özellikleri irdelenmiştir.

\section{1. $k \neq 0$ ve $m \neq 0$ için Sonuçlar}

$t \rightarrow 0$ için $\mathrm{A}(\mathrm{t})$ ve $\mathrm{B}(\mathrm{t})$ metrik potansiyelleri ve $\mathrm{C}(\mathrm{t})$ sabitken, $\rho(\mathrm{t}), \mathrm{p}(\mathrm{t}), p_{m}(t), \rho_{m}(t), \sigma_{w}(t)$ değerleri sonsuza yaklaşmaktadır. $t \rightarrow \infty$ durumunda, $\mathrm{A}(\mathrm{t}), \mathrm{B}(\mathrm{t})$ ve $\mathrm{C}(\mathrm{t})$ sonsuza yaklaşmaktadır. Ayrıca zaman arttıkça $\rho(\mathrm{t}), p_{m}(t), \rho_{m}(t), \sigma_{w}(t)$ değerleri sıfıra yaklaşmakta, $p(t)$ artmaktadir.

Bu model, $t=\frac{m s+1}{k s}$ veya $t=\frac{m s-1}{k s}$ değerlerinde tekilliklere sahiptir. Enerji yoğunluğunun ve basıncın sürekli olması için $t \neq \frac{m s+1}{k s}$ veya $t \neq \frac{m s-1}{k s}$ olması gerekir.

\section{2. $k=0$ ve $m \neq 0$ için Sonuçlar}

Evrenin başlangıcında $\mathrm{A}(\mathrm{t}), \mathrm{B}(\mathrm{t})$ metrik potansiyelleri ve $\mathrm{C}(\mathrm{t})$ skaler alan sabit değerler alırken, $\rho(\mathrm{t}), \mathrm{p}(\mathrm{t}), p_{m}(t), \rho_{m}(t), \sigma_{w}(t)$ değerleri sonsuza yaklaşmaktadır. Zaman arttıkça $\mathrm{A}(\mathrm{t}), \mathrm{B}(\mathrm{t})$ ve $\mathrm{C}(\mathrm{t})$ sonsuza yaklaşırken $\mathrm{p}(\mathrm{t}), \rho(\mathrm{t}), p_{m}(t), \rho_{m}(t)$ değerleri azalmakta ve $\sigma_{w}(t)$ sıfıra yaklaşmaktadir.

Bu modelde $t=-\frac{c_{6}}{m c_{5}}$ noktasında tekillik mevcuttur. Bu nedenle enerji yoğunluğunun ve basıncın sürekli olması için $t \neq-\frac{c_{6}}{m c_{5}}$ olmalıdır.

Sonuçları incelediğimizde $\mathrm{C}(\mathrm{t})$ skaler alanımızın her iki durum içinde sabit çıkmaktadır. $\mathrm{Bu}$ durumda (2.2) ve (2.3) denklemlerindeki $T_{i k}^{C}=0$ olacağından genel rölativiteye dönüşür. Creation Field teoride n-boyutlu Kaluza-Klein metriğinde Domain Wall madde çözümüne izin vermediği görülmektedir. 


\section{Teșekkür}

$\mathrm{Bu}$ çalışma Çanakkale Onsekiz Mart Üniversitesi, Fen Bilimleri Enstitüsü, Matematik Anabilim Dalı yüksek lisans öğrencisi olan Nur GÜLTEKİN' in Yrd. Doç. Dr. Can AKTAŞ danışmanlığında yürütülen tez çalışmasının bir parçasıdır. Bu çalışma Çanakkale Onsekiz Mart Üniversitesi Bilimsel Araştırma Proje birimi tarafından desteklenmiştir. Proje numarası: FYL2016-643

\section{Kaynaklar}

Adhav K. S., Nimkar A. S., Dawande M. V., 2008. String Cloud and Domain Walls with Quark Matter in n-dimensional Kaluza-Klein Cosmological Model. International Journal of Theoretical Physics. 47(7):2002-2010.

Adhav K. S., Gadodia P. S., Bansod A. S., 2010. Higher Dimensional Bianchi Type- I Universe in Creation Field Cosmology. International Journal of Theoretical Physics. 49: 957-966.

Adhav K., Nimkar A. S., Mete V.G., Dawande M. V., 2010. Axially Symmetric Bianchi Type- I Model with Massless Scalar Field and Cosmic Strings in Barber's Self Creation Cosmology. International Journal of Theoretical Physics. 49: 1127-1132.

Adhav K.S., Bansod A.S., Desale M.S., Raut R.B., 2011. LRS Bianchi type-I models with constant deceleration parameter in creation field cosmology. Astrophysics and Space Science. 331: 689-695.

Akarsu Ö., Dereli T., 2012.Cosmological Models with Linearly Varying Deceleration Parameter Int J. Theor. 51:612-621.

Bali R., Kumawat M., 2011. Cosmological Models with Variable $G$ in $C$-Field Cosmology. International Journal of Theoretical Physics. 50: 27-34.

Bondi H., Gold T., 1948. The Steady-State Theory of the Expanding Universe. 108: 252-270.

Efstathion G. et al., 2002. Evidence from non-zero and a low matter density from a combined analysis of the 2dF Galaxy redshift Survey and cosmic microwave background anisotropies, Mon. Not. Roy. Aston. Soc. 330: L29-35.

Hoyle F., Narlikar J. 1966. A Radical Departure from the Steady-State Concept in Cosmology. Proc. R. Soc. (Lond.) A 290: 162-167. 
Gültekin ve Aktaş, 2017

Katore S. D., Hatkar S. P., Baxi R. J., 2016. Domain Wall Cosmological Models With Deceleratıon Parameter In Modıfied Theory Of Gravitation. Chinese Journal Of Physics.

Rahaman F., Bhui B. C., Mukherji R., 2005. Global Monopole in the Presence of a C-field. Chinese Journal of Physic 43: 806-812.

Rahaman F., Kalam M., Chakraborty S., 2007. Gravitational lensing by a stable c-field wormhole. Chinese Journal of Physics. 45(5):518-529.

Singh K. P. , Singh K. M. 2014.On Bianchi Type III String Cloud Universe Containing Strange Quark Matter. Int. J. Of Astronomy and Astrophys. 4: 544-549.

Tegmark M. et.al., 2004, Cosmological parameters from SDSS and WMAP, Phys. Rev. D 69: 103501-103526.

Vilenkin A., 1981. Gravitational field of vacuum domain walls and strings. Physical Review D. 23: 852-859.

Vilenkin A., Shelleard E. P. S., 1994. Cosmic Strings and Topological Defects, Cambridge University 